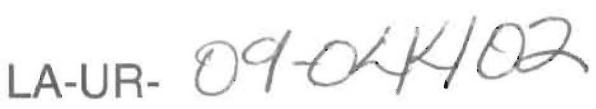

Approved for public release;

distribution is unlimited.

\author{
\begin{tabular}{l|l} 
Title: & $\begin{array}{l}\text { CHARACTERIZING THE EFFECTS OF RATCHET } \\
\text { GROWTH ON PBX } 9502\end{array}$
\end{tabular} \\ Author(s): D. G. Thompson, G. W. Brown, J. T. Mang, R. DeLuca, B. \\ Patterson and S. Hagelberg \\ Los Alamos National Laboratory, Los Alamos NM 87545 \\ Intended for: \\ Proceedings of the 16th Topical Conference on Shock \\ Compression of Condensed Matter, American Institute of \\ Physics, 28 June - 03 July 2009, Nashville, TN.
}




\title{
CHARACTERIZING THE EFFECTS OF RATCHET GROWTH ON PBX 9502
}

\author{
D. G. Thompson, G. W. Brown, J. T. Mang, R. DeLuca, B. Patterson and S. Hagelberg
}

Los Alamos National Laboratory, Los Alamos NM 87545

\begin{abstract}
Pressed composites of TATB (2,4,6-trinintro-1,3,5-benzenetriamine) undergo irreversible volume change when subjected to thermal cycling. Using micro x-ray computed tomography and ultra-small angle neutron scattering, we have characterized the micro-structure of as-pressed and ratchet grown specimens of PBX 9502, a TATB-based composite, thereby distinguishing the effects of ratchet growth from the effects of density alone. Porosity differences are shown to effect mechanical properties, presented here, with ongoing efforts to evaluate sensitivity and/or performance effects.
\end{abstract}

Keywords: PBX 9502, ratchet growth, TATB, irreversible volume change, density effects.

PACS: 62.20.M, 61.72.Qq, 61.05.fg

\section{INTRODUCTION}

TATB-based composites undergo ratchet growth, an irreversible volume expansion, upon thermal cycling. Growth is asymptotic for repeated excursions over a given thermal range, beginning anew if that range is increased. The mechanism of growth remains unknown, but likely arises from the highly anisotropic coefficient of thermal expansion (CTE) of the TATB crystal.[1,2] Ratchet-growth studies often emphasize density change alone [1-3]. In this work we show that ratchet grown density changes are characterized by pore structure and corresponding material properties indicative of unique micro-structural damage [4].

PBX 9502 is a plastic bonded explosive containing $95 \mathrm{wt} \%$ TATB and $5 \mathrm{wt} \%$ KelF binder. Four populations of Recycled PBX 9502 cylindrical cores were prepared for this study, Fig. 1. Two sets of cores were used in their "aspressed" form, to span a wide range of densities (Charges 01 and 03 spanning high and low density, respectively). Two other sets of cores came from nominal high density pressings, but were thermal cycled to lower densities prior to testing (Charge 02 and 04 were ratchet growth with 0 and 100 psi axial confinement, respectively).

From the four core populations, specimens have been machined for mechanical testing, detonation failure cones, and plate impact tests. Micro-structural characterization was performed using micro x-ray computed tomography, and ultra-small-angle neutron scattering (USANS). This paper will focus on the micro-structural characterization and some of the corresponding differences observed in the mechanical properties.

\section{EXPERIMENTAL AND RESULTS}

Four hemispherical charges of Recycled PBX 9502, lot HOL88A891-006, were isostatically pressed by heating molding powder to $110^{\circ} \mathrm{C}$, placing it in a neoprene bag with an inner mandrel, and pressurizing with 4 cycles of $5 \mathrm{~min}$. dwells. A pressure of $20 \mathrm{kpsi}$ was used for Charges 01,02 and 04 , while a pressure of $10 \mathrm{kpsi}$ was used for Charge 03 , resulting in a lower as-pressed density.

From each charge, cylindrical cores $(2.54 \mathrm{~cm}$ diameter by $110.16 \mathrm{~cm}$ long) were removed 
perpendicular to the equatorial plane, resulting in two distinct groups of core densities (Fig. 1). Higher- and lower-density cores were adjacent to the inner and outer charge cavity, respectively (Charge 04 used only the high-density cores).

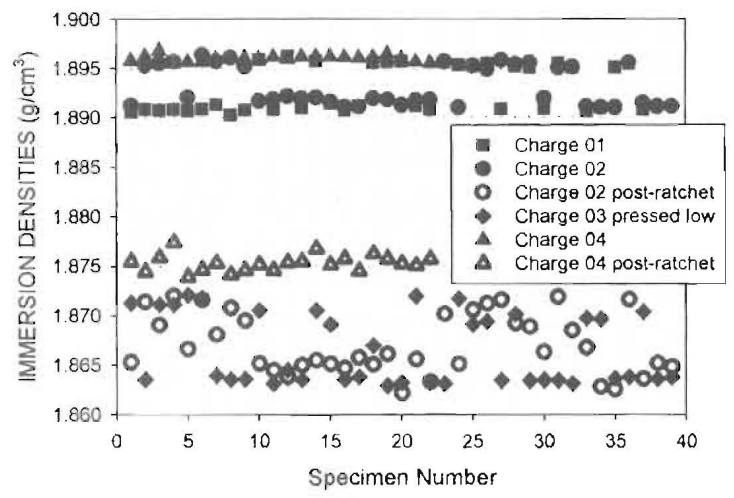

Figure 1: Immersion density values for PBX 9502 cores vs. core number. Solid symbols are density values for as-pressed densities, open symbols are density values after thermal cycling.

Tensile data are included in this paper from a previous study on nominal recycled PBX 9502, lot 891-007 (labeled "Lot 007").

The density of all cores and the subsequently machined specimens were measured using immersion methods in water and a NIST standard reference, Lot 007 specimen densities were measured using the same procedure.

Cores from Charges 02 and 04 were thermal cycled in batches ( 8 to 10 cores per batch), inside an explosion-proof Tenney Benchmaster, model BTC-SPL. The cores were placed into spring-rig steel fixtures, with 0 and 100 psi load placed on the Charge 02 and Charge 04 cores, respectively. Thermocouples were used to monitor 30 cycles of the following profile: $1^{\circ} \mathrm{C} / \mathrm{min}$ ramp from 23 to $85^{\circ} \mathrm{C}$, one hour hold, $1^{\circ} \mathrm{C} / \mathrm{min}$ ramp to $-65^{\circ} \mathrm{C}, 1$ hour hold, $1^{\circ} \mathrm{C} / \mathrm{min}$ ramp back to $23^{\circ} \mathrm{C}$.

Post-ratchet-growth densities for cores from Charges 02 and 04 are plotted in Fig. 1. The initial high-low density distribution for charge 02 cores is retained in the ratchet grown cores. Normalized average density change of Charge 02 and 04 cores was 1.38 and $1.09 \%$, respectively, anticipated from previous studies [3].
In Fig. 2, averaged dimensional changes caused by ratchet growth of Charge 02 and 04 cores are shown. Pre- and post-ratchet dimensions were hand-measured using a micrometer. Three diameters, at 1, 2 and 3 inches along the core length, and three lengths were measured and averaged. While the 100 psi confinement stopped ratchet growth in the axial direction, as stated in previous work [5], it served to increase growth laterally.

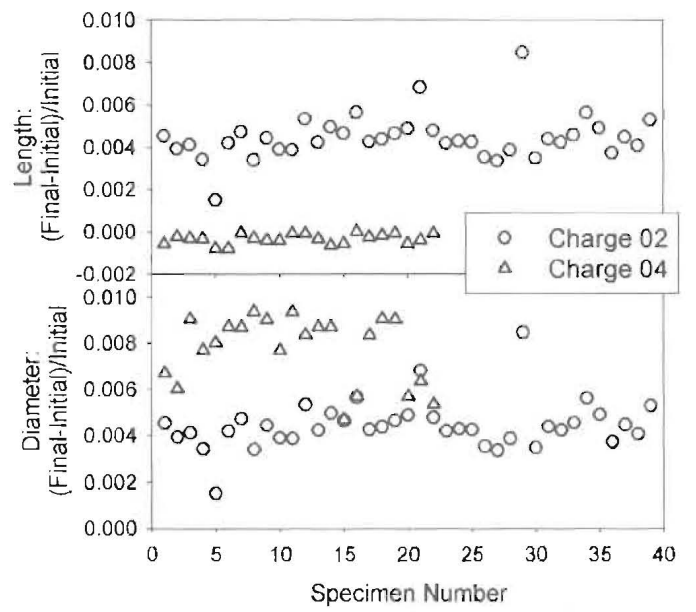

Figure 2: Specimen dimensions from pre- and postthermal cycled cores.

Quasi-static uniaxial tensile testing was performed remotely, in strain rate control, on an Instron 5567 with TestWorks control software. Strain was measured using two, oppositelymounted, knife-edge extensometers, gauge length $2.54 \mathrm{~cm}$, on tapered-end dogbone specimens. Load was measured using a $5 \mathrm{kN}$ load cell. Three tensile parameters were used to show stress-strain trends as a function of PBX 9502 specimen density: the ultimate stress (in $\mathrm{MPa}$ ), the strain value which corresponds to the ultimate stress (reported in \%), and the modulus, or slope, of the stress-strain curve at some fixed percentage of the ultimate stress (in $\mathrm{MPa} / \%)$. Using a Bemco environmental chamber, tests were performed at -20 and $50^{\circ} \mathrm{C}$, and results from the latter are shown in Fig. 3.

Brazilian disks, $20 \mathrm{~mm}$ in diameter and 5 mm thick, were also tested on the Instron. Tests were performed at $50^{\circ} \mathrm{C}, 0.02 \mathrm{in} / \mathrm{min}$. In these tests, vertical compression across the specimen diameter results in horizontal tensile failure. 
Brazilian tests parameters, plotted vs, density in Fig. 4, include maximum load, extension at maximum load, and the slope of load versus extension at $40 \%$ of the maximal load.

Tomographic images were obtained using an Xradia (Concord, CA) micro CT Instrument with an acceleration voltage of $80 \mathrm{kV}$. The instrument uses a Hamamatsu micro focus tungsten source ( $5 \mu \mathrm{m}$ nominal spot size, 40 - $150 \mathrm{kV}$ broad band) which produces a cone beam of x-rays with a $43^{\circ}$ spread. Typically, 721 x-ray radiographic images with an exposure time of 1 minute were collected with a $20 \mathrm{X}$ objective as the specimen was rotated $180^{\circ}$; total collection time was $\sim 13 \mathrm{hrs}$. Pixel size in the radiographic images with the 4 megapixel CCD camera was $0.55 \mu \mathrm{m}$.

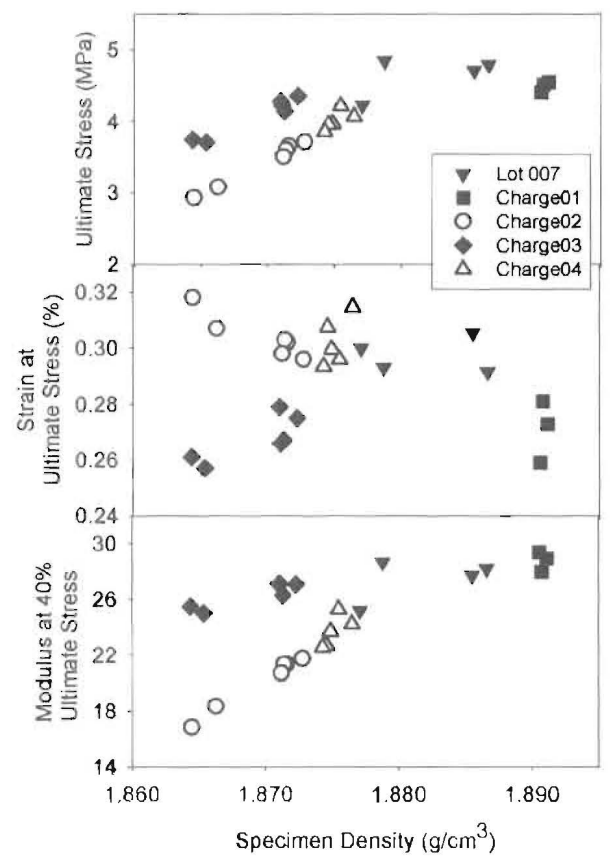

Figure 3: Reduced $50^{\circ} \mathrm{C}$ tensile parameters plotted as a function of specimen density. Filled symbols are aspressed; open symbols are thermal cycled specimens.

Reconstruction to a 3D image was performed using Xradia software. Datasets were binned by a factor of 2 in every dimension producing a 1 gigavoxel data set with voxel size of $1.10 \mu \mathrm{m}$. Four specimens (each $\sim 0.04 \mathrm{~g}$ ) were imaged, two from Charge 02 and two from Charge 03 .

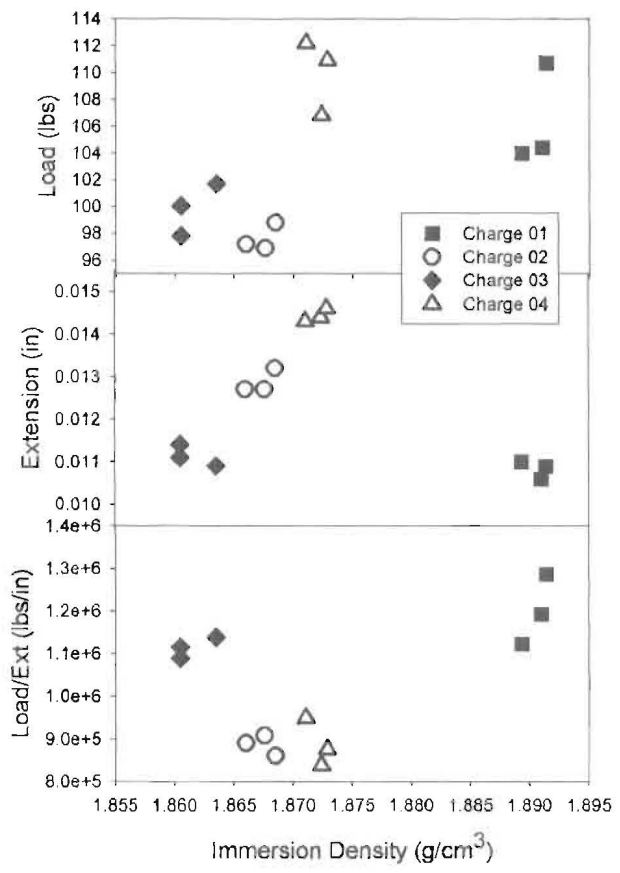

Figure 4: Reduced $50^{\circ} \mathrm{C}$ Brazilian disk parameters plotted vs. specimen density. Symbols same as Fig. 3.

Image analysis was performed using Clemex software. The results of three different grayscale thresholds were averaged for each of 3 slices from each sample, plotted in Fig. 5.

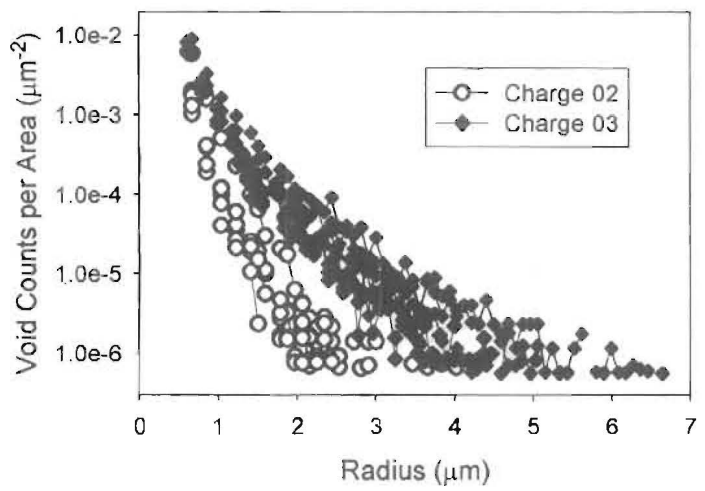

Figure 5: Pore-size distribution from Clemex software analysis of $\mathrm{x}$-ray tomography images (see text).

USANS measurements [6] were performed at the National Institute of Standards and Technology Center for Neutron Research 
(NCNR) using the BT-5 Perfect Crystal SANS instrument and a monochromatic neutron wavelength of $2.4 \AA$. Samples were sealed inside rectangular cells made of fused silica. The BT-5 instrument is a Bonse-Hart camera [7] with a maximum effective Q-range of $0.00003-0.01 \AA^{-1}$ and size range from $0.1-20 \mu \mathrm{m}$. Analysis assumed spherical voids to calculate void volume distributions, Fig. 6.

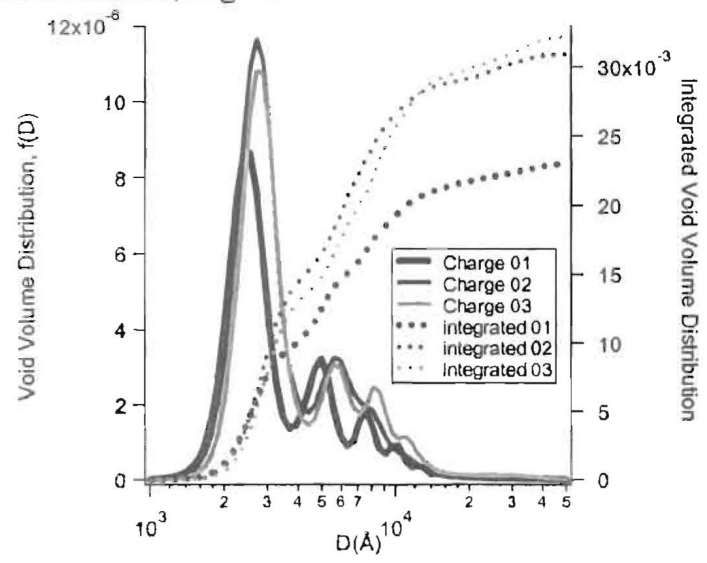

Figure 6: Pore-size distribution from USANS (see text).

\section{DISCUSSION}

Tensile parameters, Fig. 3, clearly show that for material of equivalent density, ratchet grown specimens have reduced strength and modulus, consistent with damage accumulation, and increased strain capacity, when compared with the as-pressed specimens. Uniaxial tensile trends do not distinguish the behavior of confined vs. unconfined ratchet grown specimens. Quite similar behavior was observed in quasi-static compression, except that maximum compressive strength correlated linearly with density, regardless of how that density was obtained (data not shown). Brazil disk response for the unconfined specimens, Charge 02, are similar to the response observed in unaxial tension, however, the confined specimens, Charge 04, fall on different trend lines in the Brazil data. This is likely due to the more complex stress state in the latter test, coupled with the anisotropy of the damage in the Charge 04 specimens.

Micro X-ray CT and USANS data cover the same range of pore size and give similar information showing that for specimens of similar density, ratchet growth results in smaller pores, more homogeneously distributed, where as-pressed parts have fewer and larger pores.

Micro-structural characterization of Charge 04 specimens is underway, and we anticipate some observable anisotropy in the pore structure. Additional tests have been initiated using the cores described in this paper, including plate impact tests (SDT), detonation failure cones, and cylinder tests.

\section{ACKNOWLEDGEMENTS}

Funding was provided by the Weapons Surety program at LANL. LANL is managed and operated by LANS, LLC, under DOE/NNSA contract DE-AC52-06NA25396.

\section{REFERENCES}

1. Kolb, R., and Rizzo, H. F., "Growth of 1, 3, 5Triamino-2, 4, 6-trinitrobenzene (TATB), I. Anisotropic Thermal Expansion," Prop, Explos., and Pyrotech., 1979, $4,10$.

2. Rizzo, H. F., Humphrey, J. R., and Kolb, J. R., "Growth of 1,3,5-Triamino-2,4,6-Trinitrobenzene (TATB), Control of Growth by Use of High $\mathrm{T}_{\mathrm{g}}$ Polymeric Binders," UCRL-52662, 1979, Lawrence Livermore National Laboratory, Livermore, CA, USA.

3. Cunningham, B., Andreski, B., "Effects of Thermal Cycling on the Tensile Strength of PBX 9502," $25^{\text {th }}$ Aging, Compatibility and Stockpile Stewardship Conference, Livermore, 18 - 20 Nov 2003, LLNL, Livermore, CA 2003.

4. Thompson, D.G., et al., submitted to PEP, 2009.

5. Benziger, T. M. and Urizar, M. J., WX-2 Monthly Reports, 1974, Los Alamos Scientific Laboratory, Los Alamos, NM.

6. Glatter, O. and Kratky, O., Small Angle X-ray Scattering, Academic Press, London, 1982.

7. Barker, J. G., Glinka, C. J., Moyer, J. J., Kim, M. H., Drews, A. R., and Agamalian, M., J. Appl. Cryst, $1995,38,1004$.

8. Willey, T. M., Buuren, T. v., Lee, J. R. I., Overturf, G. E., Kinney, J. H., Handly, J., Weeks, B. L., Ilavsky, "Changes in Pore Size Distribution upon Thermal Cycling of TATB-based Explosives Measured by UltraSmall Angle X-Ray Scattering," J., Prop, Explos., and Pyrotech., 2006, 6, 466. 